\title{
Incremental Prognostic Value of Echocardiographic Strain and Its Association with Mortality in Cancer Patients
}

Isaac B. Rhea $\mathrm{MD}^{1}$, Sarada Uppuluri MD², Stephen Sawada MD FACC ${ }^{1}$, Bryan P. Schneider MD ${ }^{3}$, Harvey Feigenbaum MD FACC $^{1}$

\section{Abstract}

\section{Background}

Left ventricular global longitudinal systolic strain (GLS) has been shown to be superior to ejection fraction (EF) in detecting subclinical dysfunction in cancer patients and predicting mortality in patients with cardiovascular disease. Cancer-related fatigue is common in the later stages of neoplastic malignancies and may be indicative of non-overt heart failure. In this study we sought to determine whether reduced strain by echocardiography was associated with all-cause mortality in a cancer cohort.

\section{Methods}

In this retrospective study, one hundred and twenty patients with cancer undergoing or scheduled to undergo chemotherapy and with a normal EF (> 50\%) had assessments of GLS. GLS was derived by averaging all speckle tracking strain segments of the left ventricle.

Results

Over an average follow-up of $21.6 \pm 13.9$ months, 57/120 patients died. Univariate predictors of all-cause mortality $(p<0.10)$ were Eastern Cooperative Oncology Group Performance Status (ECOGPS), male sex, hematologic malignancy, beta blocker usage, and GLS. Multivariate analysis of all significant univariate variables showed that ECOGPS ( $p<0.001$, HR 2.12 [1.54-2.92]), male sex ( $p=0.014$, HR 1.93 [1.14-3.27]), and GLS ( $p=0.012, H R 0.89$ [0.81-0.97]) were significant ly and independently associated with mortality. Stepwise analysis of the multivariate associations showed an increase in global $\chi^{2}$ after adding GLS ( $\left.p=0.011\right)$ to significant clinical variables.

\section{Conclusions}

ECOGPS, male sex, and GLS were significantly associated with all-cause mortality in cancer patients with normal EFs receiving chemotherapy. Adding GLS to significant clinical variables provided incremental prognostic information.

Keywords: Strain, Mortality, Neoplasia, Echocardiography, Speckle-tracking imaging, Prognosis

\section{Introduction}

Left ventricular ejection fraction (EF) has long been considered the standard for assessing cardiac function in most cardiac disorders and in monitoring chemotherapy-induced cardiotoxicity. However, EF rarely declines to a measurable degree from some chemotherapy agents (i.e. anthracyclines) until irreversible and symptomatic damage has been done.[1] Strain echocardiography (SE) is an emerging technique that has been shown to be clinically useful due to its ability to detect myocardial dysfunction earlier and with greater sensitivity than EF or wall motion analysis. Left ventricular global longitudinal systolic strain (GLS) has been demonstrated to be a significant predictor of chemotherapyinduced cardiotoxicity in patients with preserved EF.[2] It has also been demonstrated to be a predictor of multiple outcomes including all-cause mortality in patient populations with a strong prevalence of cardiac disease.[3-7] This

This is the author's manuscript of the article published in final edited form as:

Rhea, I. B., Uppuluri, S., Sawada, S., Schneider, B. P., \& Feigenbaum, H. (2015). Incremental Prognostic Value of Echocardiographic Strain and Its Association With Mortality in Cancer Patients. Journal of the American Society of Echocardiography, 28(6), 667-673. http://doi.org/10.1016/j.echo.2015.02.006 
suggests that reduced strain is associated with death from cardiac disease. However, SE has not been investigated for its potential to predict death independently of underlying cardiac disease.

Since cancer-related fatigue (CRF) worsens as cancer progresses, it stands to reason that some factors responsible for CRF may correlate with mortality in cancer patients. [8] Schünemann et al noted that CRF may be associated with subclinical cardiac dysfunction.[9] Thus, we investigated whether mortality in cancer patients without excess cardiac disease was associated with reduced cardiac function on SE.

We compared the association of strain by SE with mortality against clinical performance status which is a validated measure of determining prognosis across multiple cancer types.[10-13]

\section{Methods}

\section{Study Population}

This study was approved by the Indiana University Institutional Review Board. We queried the echocardiography database (Fuji Synapse 4.0 Indianapolis, IN) for patients with cancer who had echocardiograms with strain measurements performed in relation to chemotherapy or bone-marrow transplants. Per laboratory policy, if possible strain was calculated at the time of echocardiogram for all studies ordered either as standard cardiac monitoring for chemotherapy regiments and in symptomatic patients with concern of chemotherapy-related cardiac toxicity. We identified 138 patients from 2009-2011 cared for at the Indiana University Simon Cancer Center who had both 2D echo images and pre-existing speckle tracking polar plots calculated directly on the instrument consoles. We excluded patients with known systolic dysfunction (14 patients with LVEF $<50 \%$ and one patient on inotropic therapy) and patients with overall poorly tracking strain studies $(n=3)$. The study population was thus comprised of 120 retrospectively identified patients with a history of recent or imminent chemotherapy, a normal EF, and previously acquired strain images. Nine patients obtained their study before ever receiving chemotherapy for their active malignancy.

\section{Clinical Data Methods}

The clinical history was obtained via chart review in which the type of malignancy, age at time of last visit/death, sex, beta blocker usage, ECOGPS, and various comorbidities were documented from approximately the time the echo was performed. Any past or imminent exposures to anthracyclines were recorded. Eastern Cooperative Oncology Group Performance Status (ECOGPS) and Karnofsky Performance Status (KPS) are widely validated tools for measuring performance status and are often used to predict mortality for multiple cancer types.[11] ECOGPS was taken from oncology clinic/consult notes. See Table 1 for clinical descriptions of the different ECOGPS grades.

For cases in which only KPS was available, the value was converted to an ECOGPS grade as in equation 1. Four patients without distinct documentation of performance status had notes that heavily implied no functional limitations and were counted as an ECOGPS of 0.

Equation 1. Rounddown $\left(5-\frac{K P S}{20}\right)=$ ECOGPS

To determine if cancer was a likely cause of death, all patients that died were analyzed via chart review for the presence of advanced (i.e. potentially rapidly fatal) cancer and for any clinical indications of subsequent cardiotoxicity defined as an absolute drop in $\mathrm{EF}>10 \%$ to a level below $50 \%$ at the time of the last echo not readily explained by another acute process. 
Two-dimensional echocardiographic measurements were performed using a Vivid 7 or Vivid Q echograph (General Electric Medical Systems, Milwaukee, WI). The echocardiographic examinations were performed according to ASE guidelines.[14] The images were recorded digitally and analyzed off line (Fuji Synapse 4.0 Indianapolis, IN).

\section{Strain Acquisition}

The strain measurements were obtained by experienced sonographers using a Vivid 7 and Vivid Q echograph (General Electric Medical Systems, Milwaukee, WI) at the same time that the standard 2D echocardiograms were performed. To make the speckle tracking strain calculations, apical 4, 3, and 2-chamber views were stored in a cine loop format. Images were obtained between 50-70 frames per second. The analysis was made at the conclusion of the examinations using a propriety software package on the instrument. The software used requires the operator to identify three points on each view (the two mitral annulus attachments to the myocardium and also the apex). The software then automatically determines the strain measurements in the six segments of each view. The calculated peak strain values for the entire myocardium can then be displayed as a 17 or 18 segment polar plot, or "bull's eye" diagram. The software calculates an average strain value for each view individually then a global average incorporating all three (Figure 1).

GLS measurements were taken directly from the polar plot screen without adjustment. Those echocardiograms in which a polar plot could not be constructed were not considered. A high threshold was maintained for excluding any remaining strain echocardiograms based on strain calculations which appeared grossly inaccurate, and only one strain echocardiogram was excluded by this criterion. In this case 8 of 18 contiguous segments were calculated as either expanding during systole or non-tracking yet without any echocardiographic or clinical findings to substantiate a regional dysfunction.

\section{Reproducibility}

As our institution does not have the capability to retrospectively collect strain reproducibility data using the native GE software, we used data we had prospectively collected from a general cohort of patients that had echocardiograms ordered and performed at roughly the same time as the study patients. Inter-observer and intra-observer variability were measured using different cohorts of patients ( $n=36$ for both) with various pathologies and with $E F>50 \%$. These studies had repeat strain analyses performed by the same sonographer or by a different sonographer for the purposes of determining reproducibility.

\section{Outcomes}

All-cause mortality was the primary end point. Follow-up was obtained by using the patient's hospital and clinic charts, online obituary records, or the Social Security Death Index.

\section{Statistical Analysis}

Analysis was carried out using SPSS (version 16, SPSS Inc., Chicago, III). To simplify analysis and communication, all strain values were multiplied by -1 such that greater positive strain values refer to greater myocardial contraction and viceversa.[15] The $\chi^{2}$ test was used to determine significant differences between 2 groups of categorical or continuous variables.

Univariate binary logistic regression was performed to determine if variables which might affect strain such as age, sex, time from chemotherapy to echo, prior thoracic radiation, prior anthracycline use, hypertension, coronary artery disease, or diabetes mellitus were independently associated with an abnormal GLS defined as $<18$. 
Survival was assessed using Kaplan-Meier analysis and significant differences in survival were assessed with log-rank test. Cox proportional hazards analysis was used to determine significant univariate factors associated with all-cause mortality. Two different types of multivariate analyses were performed. The initial analysis was conducted with a forward selection method including all clinical and echocardiographic variables with $p<0.10$ on univariate analysis. Variables with $p<0.05$ on multivariate analysis were considered significantly and independently associated with mortality. A second stepwise analysis was performed using the clinical variables that were independently associated with mortality in the first step and SE variables in the second step to determine if strain variables added incremental prognostic value. Reproducibility was reported as the average percent measurement difference/mean.

\section{Results}

Table 2 contains the clinical and echocardiographic information for the population. Table 3 provides a breakdown of the malignancies. There are 122 malignancies as two patients had two simultaneous primary cancers. Only 4 patients (3\%) had significant coronary disease. One of the surviving patients had minimal records available after leaving the country and thus was the only survivor with $<14$ months of follow-up. Aside from one patient with mild-to-moderate valvular disease, all patients had no more than mild valvular disease.

Over $21.6 \pm 13.9$ months of follow-up, there were $57 / 120$ deaths (48\%). One patient died from heart failure thought to be due primarily to chemotherapy induced cardiotoxicity, and one patient died abruptly without signs of cardiotoxicity or an active malignancy at last follow-up. The remaining 55 patients had advanced cancer at last follow-up prior to death.

Binary logistic regression showed that among the risk factors of age, sex, time from chemotherapy to echo, prior thoracic radiation, prior anthracycline use, hypertension, coronary artery disease, and diabetes mellitus, only HTN $(p=0.064)$ and CAD $(p=0.009)$ had $p$ value significance $<0.10$ associated with a decreased GLS.

Table 4 shows the results of univariate and multivariate analyses of all clinical and echocardiographic variables. In this set of variables the significant univariate factors associated with mortality $(p<0.1)$ were male sex, ECOGPS, hematologic malignancy, beta blocker usage, and GLS. Neither prior anthracycline use nor prior thoracic radiation was associated with mortality. On multivariate analysis of the significant univariate associated factors, male sex, ECOGPS, and GLS were independently associated with mortality.

A second multivariate analysis was done starting with the significant independent clinical variables (male sex and ECOGPS). GLS was added via stepwise block analysis to these clinical parameters and was found to be significant $(p=0.011)$ as detailed in Figure 2.

Figure 3 demonstrates the Kaplan-Meier survival curve comparing abnormal GLS $(<18)$ to normal GLS ( $\geq 18)$. Of note $48 \%$ of the patients with abnormal GLS (25/52) died within 12 months of the strain echo compared with only $24 \%$ of the group with normal GLS (16/68).

The interobserver and intraobserver reproducibility for our facility is $7.3 \%$ and $5.8 \%$ for GLS respectively and $7.7 \%$ and $8.7 \%$ for EF respectively.

\section{Discussion}

The present study demonstrates that GLS is independently associated with mortality in cancer patients with preserved EF and adds incremental prognostic value when added to clinical variables.

Strain as a nonspecific marker of prognosis in cancer patients 
While the specific causes of death for the study population are unknown, it is reasonable to assume that an overwhelming majority of patients died primarily from their malignancies as opposed to cardiotoxicity as all but two were noted to have advanced symptomatic cancer at the time of their last contact with our facility prior to death. Limat et al found that among 147 lymphoma patients treated with aggressive cardiotoxic therapy only 3 died from early cardiotoxicity (within 1 year).[16] Given that our sample population did not have a significantly elevated rate of known hypertension or cardiac disease, a normal initial EF, and a normal mean wall thickness, it is reasonable to think that cardiac disease would be a lesser contributor to the high mortality in this group. Also, given that this cohort was exposed to a wide range of treatment modalities, it is not surprising that only one patient had definitive cardiac death from cardiotoxicity. Our data thus implies strain detects changes in cardiac function caused by certain forms of severe illness. The pathophysiology for this process is unknown. Although at least a portion of this phenomenon is believed to be a direct result of chemotherapy, the lack of significance of anthracycline exposure as a univariate factor associated with mortality and the lack of association with reduced GLS argues against this. One possible explanation is the increased amount of inflammatory cytokines. Severe cancer has been known to cause systemic "B-type symptoms" such as fever, chills, fatigue, and cachexia. CRF and cachexia are both associated with elevated inflammatory cytokines.[17-20] In addition, cachexia has been noted to cause myocardial dysfunction in animal models.[21] High levels of inflammatory cytokines are associated with a poor prognosis in cancer patients and with worsening cardiac disease including systolic dysfunction in more general cohorts. $[22,23]$ Thus, patients with a poor prognosis may have worse GLS due to increased inflammatory cytokines. It is currently unknown which cytokines may be most influential, but TNF $\alpha$, CRP, and IL-6 are frequently documented in both cardiac dysfunction and cachexia.[19, 20, 22] In other words, perhaps SE detects cancerinduced cardiac stress, a phenomenon that should worsen as cancer progresses. In the event this process is accurate, GLS would be an indicator of disease burden in cancer patients.

Another possible mechanism by which decreased strain may be associated with mortality in this population is due to increased autonomic dysfunction. Many studies have been published showing the high incidence of autonomic dysfunction in cancer patients. $[8,24,25]$ The most frequently discussed are cardiovascular consequences of this process such as orthostatic hypotension and loss of heart rate variability The severity of autonomic dysfunction has been correlated with time to death in cancer patients in several studies. Given that autonomic dysfunction can cause myocardial dysfunction, perhaps this too is a mechanism by which decreased strain correlates with mortality.[26] This is likely through a phenomenon of overriding adrenergic activity which is also a well-known component of heart failure. $[27,28]$ Increased adrenergic activity is common in cancer patients and is even suggested to be a cause of weight loss in these patients. [29] Cramer et al recently demonstrated how cardiovascular function was impaired in colorectal cancer patients regardless of whether or not they were receiving chemotherapy.[30] In their analysis they also noted how the heart rate variability was reduced and similar to that seen in patients with CHF.

Strain has been shown to be an excellent predictor of cardiac death in patients with pre-existing cardiac disease, but it has not been studied for its potential to predict non-cardiac death. Given the maladaptive systemic processes associated with end-stage cancer during the last few months of life, it can be difficult to call any non-traumatic death in these patients as unrelated to the existing malignancy regardless of the organ or organs whose failure are the ultimate cause of death. A potential exception to this would be organ failure and death as a result of drug toxicity. Agents such as trastuzumab, sunitinib, and doxorubicin all can cause cardiac dysfunction and clinical heart failure through direct or indirect means, but it is highly unlikely for such an agent to cause fatal heart failure over a rapid course during our monitoring period and go unrecognized. Another possible method of cardiac death in this population is sudden cardiac death as a result of a drug-induced arrhythmia. Typically sudden cardiac death is defined as a rapid and unexpected death from cardiac causes with or without known underlying heart disease.[31] In patients with advanced cancer and a poor life expectancy, sudden cardiac death is arguably impossible since the death is never unexpected. Myocardial infarction is another potential cause of rapid death in this population and is typically associated with agents such as 5-FU 
and Cisplatin, but it also has very low incidence and typically occurs shortly after drug administration and would thus not likely go unrecognized. Thus, it is a fairly safe assumption that nearly all of the deaths in our study population were noncardiac in nature. Aside from the one known patient that died from cardiotoxicity, there was only one other patient who died that did not appear to be approaching end-stage cancer at the time of his last visit. While having an exact cause of death for this patient may be preferable, it would have little impact on this study.

On univariate analysis beta-blocker use appeared to be associated with mortality but it was not independently associated with mortality on multivariate analysis. Beta-blockers are frequently used to treat cardiotoxicity, so their use in this population being associated with mortality is curious. Most likely, with a $p=0.046$ this could be entirely due to chance particularly in light of the number of variables analyzed. With such a low prevalence of usage and questionable significance it is difficult to draw firm conclusions.

\section{Limitations}

The present study deserves several comments. Cancer subtypes are not homogenous in their pathology and their systemic effects vary between subtypes. As such, it is not ideal to group different types of cancer together for analysis. Our database only had access to 120 eligible patients with both cancer and strain measurements and thus further group delineations by cancer type, specific chemotherapy agents and cumulative dosage, and the timing of the echo on the outcome were not possible to produce significantly powerful statistics. This study was not designed to have an impact on the management of oncology patients, but to test the hypothesis that some noncardiac diseases may influence strain proportionally to their severity.

This is a retrospective study performed at a tertiary referral center. There is no offline strain calculation software at our institution so all strain analyses had to be done by the sonographers on the instruments at the time of the echo before moving the study to offline storage. There is likely some degree of selection bias since not every patient at our institution with cancer had an echo study with strain. This is particularly true for those that were treatment-naïve. Also, our sonographers are encouraged to abort any efforts in acquiring strain if they notice the tracking quality to be poor. In most other cancer patients, a MUGA scan or an echo without strain may be obtained instead of a strain echo due to preference, convenience, level of experience, or the availability of strain-capable instruments. The oncologists are responsible for ordering exams for their patients and they have different criteria for ordering an echo over another imaging modality when they feel it is indicated. Of note, all of these exams were performed before the recent cardiotoxicity monitoring consensus statement was published. [33]

In spite of the limitations of the study the results suggest that a single SE performed at some point in the course of cancer treatment provides incremental prognostic information when combined with patient sex and performance status.

\section{Acknowledgements}

The authors would like to thank Dr. Daniel Rushing and Dr. Ana Barac for their assistance in finalizing this manuscript.

\section{Disclosures}

The authors do not have any relationships with industry to disclose. 


\section{References}

[1] Khouri MG, Douglas PS, Mackey JR, Martin M, Scott JM, Scherrer-Crosbie M, et al. Cancer therapy-induced cardiac toxicity in early breast cancer: addressing the unresolved issues. Circulation. 2012;126:2749-63.

[2] Thavendiranathan P, Poulin F, Lim KD, Plana JC, Woo A, Marwick TH. Use of myocardial strain imaging by echocardiography for the early detection of cardiotoxicity in patients during and after cancer chemotherapy: a systematic review. J Am Coll Cardiol. 2014;63:2751-68.

[3] Stanton T, Leano R, Marwick TH. Prediction of all-cause mortality from global longitudinal speckle strain: comparison with ejection fraction and wall motion scoring. Circ Cardiovasc Imaging. 2009;2:356-64.

[4] Sarvari SI, Gjesdal O, Gude E, Arora S, Andreassen AK, Gullestad L, et al. Early postoperative left ventricular function by echocardiographic strain is a predictor of 1-year mortality in heart transplant recipients. J Am Soc Echocardiogr. 2012;25:1007-14.

[5] Ersboll M, Valeur N, Mogensen UM, Andersen MJ, Moller JE, Velazquez EJ, et al. Prediction of all-cause mortality and heart failure admissions from global left ventricular longitudinal strain in patients with acute myocardial infarction and preserved left ventricular ejection fraction. J Am Coll Cardiol. 2013;61:2365-73.

[6] Bjork Ingul C, Rozis E, Slordahl SA, Marwick TH. Incremental value of strain rate imaging to wall motion analysis for prediction of outcome in patients undergoing dobutamine stress echocardiography. Circulation. 2007;115:1252-9.

[7] Kalam K, Otahal P, Marwick TH. Prognostic implications of global LV dysfunction: a systematic review and metaanalysis of global longitudinal strain and ejection fraction. Heart. 2014;100:1673-80.

[8] Stone CA, Kenny RA, Nolan B, Lawlor PG. Autonomic dysfunction in patients with advanced cancer; prevalence, clinical correlates and challenges in assessment. BMC Palliat Care. 2012;11:3.

[9] Schunemann M, Anker SD, Rauchhaus M. Cancer fatigue syndrome reflects clinically non-overt heart failure: an approach towards onco-cardiology. Nat Clin Pract Oncol. 2008;5:632-3.

[10] Krishnan M, Temel JS, Wright AA, Bernacki R, Selvaggi K, Balboni T. Predicting life expectancy in patients with advanced incurable cancer: a review. J Support Oncol. 2013;11:68-74.

[11] Oken MM, Creech RH, Tormey DC, Horton J, Davis TE, McFadden ET, et al. Toxicity and response criteria of the Eastern Cooperative Oncology Group. Am J Clin Oncol. 1982;5:649-55.

[12] Ploquin A, Olmos D, Ferte C, Cassier PA, Kramar A, Duhamel A, et al. Life-expectancy of patients enrolled in phase 1 clinical trials: a systematic review of published prognostic models. Crit Rev Oncol Hematol. 2012;83:242-8.

[13] Sutradhar R, Seow H, Earle C, Dudgeon D, Atzema C, Husain A, et al. Modeling the longitudinal transitions of performance status in cancer outpatients: time to discuss palliative care. J Pain Symptom Manage. 2013;45:726-34.

[14] Lang RM, Bierig M, Devereux RB, Flachskampf FA, Foster E, Pellikka PA, et al. Recommendations for chamber quantification: a report from the American Society of Echocardiography's Guidelines and Standards Committee and the Chamber Quantification Writing Group, developed in conjunction with the European Association of Echocardiography, a branch of the European Society of Cardiology. J Am Soc Echocardiogr. 2005;18:1440-63.

[15] Feigenbaum H, Mastouri R, Sawada S. A practical approach to using strain echocardiography to evaluate the left ventricle. Circ J. 2012;76:1550-5.

[16] Limat S, Demesmay K, Voillat L, Bernard Y, Deconinck E, Brion A, et al. Early cardiotoxicity of the CHOP regimen in aggressive non-Hodgkin's lymphoma. Ann Oncol. 2003;14:277-81.

[17] Bower JE, Ganz PA, Irwin MR, Castellon S, Arevalo J, Cole SW. Cytokine genetic variations and fatigue among patients with breast cancer. J Clin Oncol. 2013;31:1656-61.

[18] Burney BO, Hayes TG, Smiechowska J, Cardwell G, Papusha V, Bhargava P, et al. Low testosterone levels and increased inflammatory markers in patients with cancer and relationship with cachexia. J Clin Endocrinol Metab. 2012;97:E700-9.

[19] Fearon KC, Glass DJ, Guttridge DC. Cancer cachexia: mediators, signaling, and metabolic pathways. Cell Metab. 2012;16:153-66.

[20] Saligan LN, Kim HS. A systematic review of the association between immunogenomic markers and cancer-related fatigue. Brain Behav Immun. 2012;26:830-48.

[21] Xu H, Crawford D, Hutchinson KR, Youtz DJ, Lucchesi PA, Velten M, et al. Myocardial dysfunction in an animal model of cancer cachexia. Life Sci. 2011;88:406-10.

[22] Kalogeropoulos AP, Georgiopoulou VV, Butler J. From risk factors to structural heart disease: the role of inflammation. Heart Fail Clin. 2012;8:113-23. 
[23] Proctor MJ, Morrison DS, Talwar D, Balmer SM, O'Reilly DS, Foulis AK, et al. An inflammation-based prognostic score (mGPS) predicts cancer survival independent of tumour site: a Glasgow Inflammation Outcome Study. Br J Cancer.

2011;104:726-34.

[24] Chiang JK, Koo M, Kuo TB, Fu CH. Association between cardiovascular autonomic functions and time to death in patients with terminal hepatocellular carcinoma. J Pain Symptom Manage. 2010;39:673-9.

[25] Fadul N, Strasser F, Palmer JL, Yusuf SW, Guo Y, Li Z, et al. The association between autonomic dysfunction and survival in male patients with advanced cancer: a preliminary report. J Pain Symptom Manage. 2010;39:283-90.

[26] Triposkiadis F, Karayannis G, Giamouzis G, Skoularigis J, Louridas G, Butler J. The sympathetic nervous system in heart failure physiology, pathophysiology, and clinical implications. J Am Coll Cardiol. 2009;54:1747-62.

[27] Mancia G. Sympathetic activation in congestive heart failure. Eur Heart J. 1990;11 Suppl A:3-11.

[28] Packer M. The development of positive inotropic agents for chronic heart failure: how have we gone astray? J Am

Coll Cardiol. 1993;22:119A-26A.

[29] Hyltander A, Korner U, Lundholm KG. Evaluation of mechanisms behind elevated energy expenditure in cancer patients with solid tumours. Eur J Clin Invest. 1993;23:46-52.

[30] Cramer L, Hildebrandt B, Kung T, Wichmann K, Springer J, Doehner W, et al. Cardiovascular Function and Predictors of Exercise Capacity in Patients With Colorectal Cancer. J Am Coll Cardiol. 2014;64:1310-9.

[31] Mann DL, Zipes DP, Libby P, Bonow RO, Braunwald E. Braunwald's heart disease : a textbook of cardiovascular medicine. 10th edition. ed. Philadelphia, PA: Elsevier/Saunders; 2015.

[32] Smith Sehdev AE, Hutchins GM. Problems with proper completion and accuracy of the cause-of-death statement. Arch Intern Med. 2001;161:277-84.

[33] Plana JC, Galderisi M, Barac A, Ewer MS, Ky B, Scherrer-Crosbie M, et al. Expert Consensus for Multimodality Imaging Evaluation of Adult Patients during and after Cancer Therapy: A Report from the American Society of Echocardiography and the European Association of Cardiovascular Imaging. J Am Soc Echocardiogr. 2014;27:911-39. 

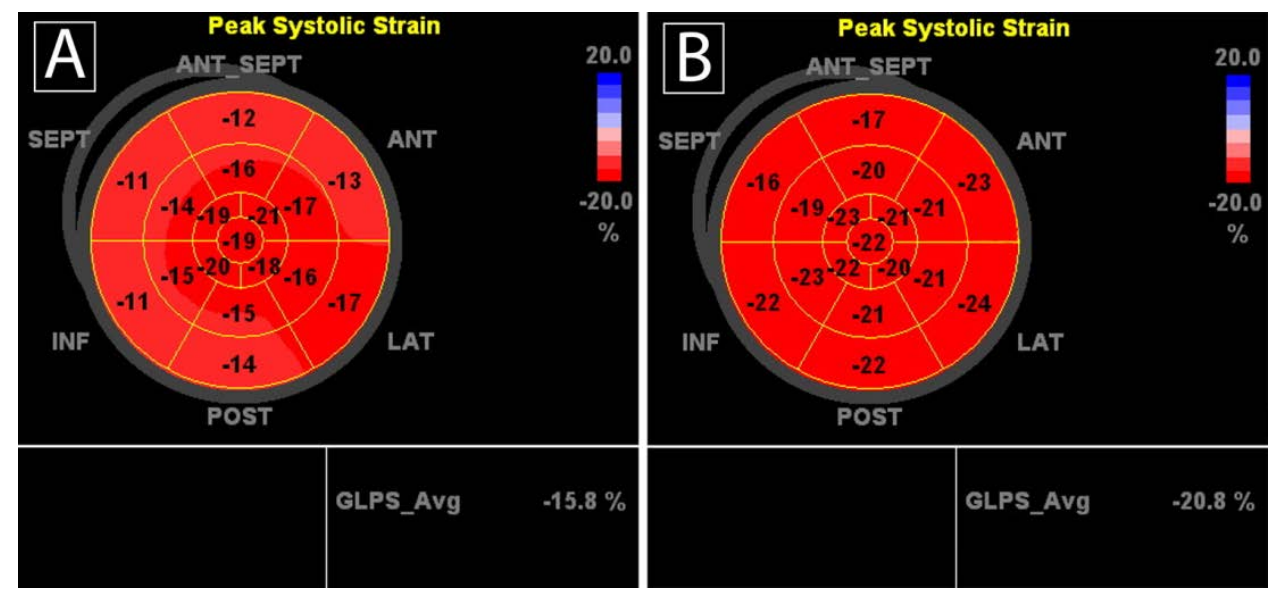

Figure 1. Sample strain polar plots for (A) a patient with abnormally low GLS (15.8, normal $\geq 18)$ who died within 12 months of this study and for (B) a patient with normal GLS (20.8) who did not die within the follow-up period of over 26 months. High strain values (most negative) are colored deep red. Lower values (less negative) are lighter red and reversed strain values (positive, not pictured) are shades of blue. Note that the strain values have been multiplied by $(-1)$ in the article text to simplify descriptive language. GLPS_Avg = left ventricular global longitudinal systolic strain. 


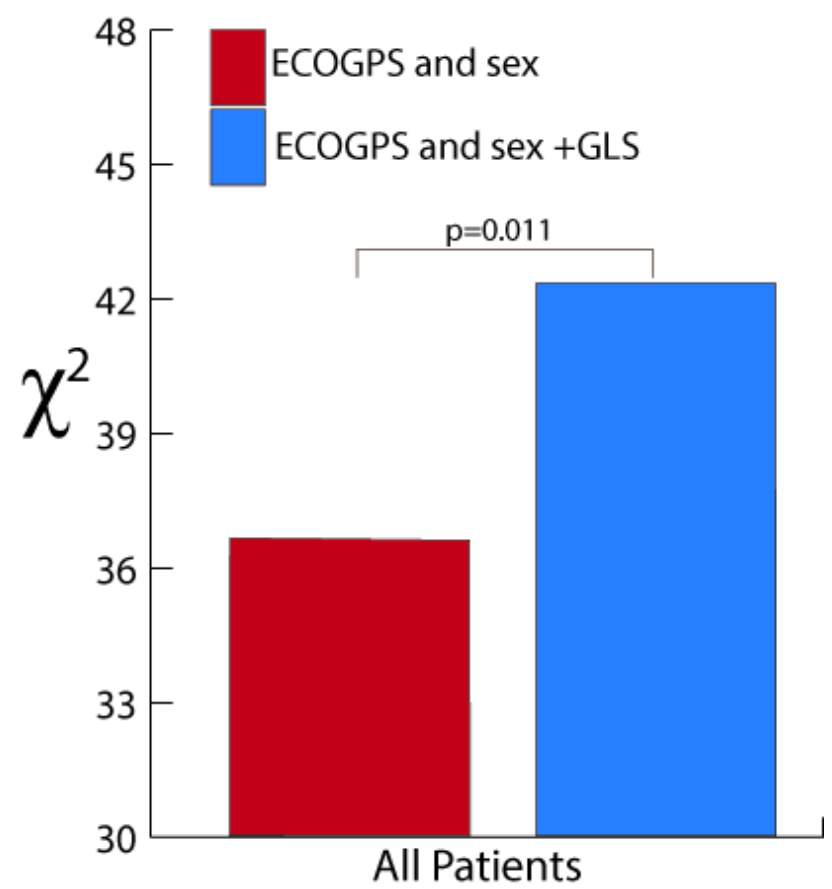

Figure 2. Stepwise addition of GLS to the clinical independent mortality variables. On multivariate stepwise block analysis, adding GLS to the clinical independent mortality variables (ECOGPS and sex) gives significant additional prognostic value. GLS = left ventricular global longitudinal systolic strain. 


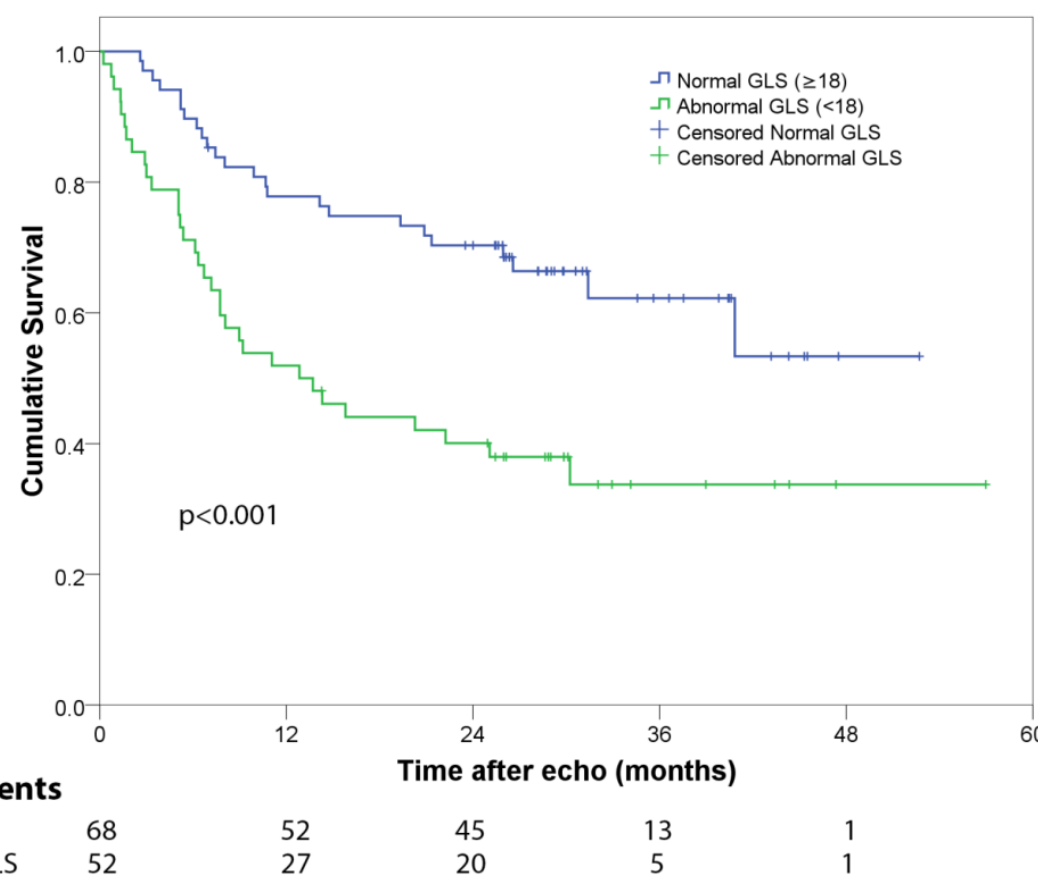

Figure 3. Kaplan-Meier curves for normal and abnormal GLS.

Separating all patients into normal $(\geq 18)$ and abnormal $(<18)$ GLS gives two groups with significant differences in mortality, particularly at 6-12 months. Remaining patients in each group are listed at $0,12,24,36$, and 48 months. GLS = left ventricular global longitudinal systolic strain 
Table 1. ECOGPS grades[11]

\begin{tabular}{|c|c|}
\hline ECOGPS Grade* & Description \\
\hline 0 & Fully active, able to carry on all pre-disease performance without restriction \\
\hline 1 & $\begin{array}{l}\text { Restricted in physically strenuous activity but ambulatory and able to carry out work of a light or } \\
\text { sedentary nature, e.g., light house work, office work }\end{array}$ \\
\hline 2 & $\begin{array}{l}\text { Ambulatory and capable of all self-care but unable to carry out any work activities. Up and about } \\
\text { more than } 50 \% \text { of waking hours }\end{array}$ \\
\hline 3 & Capable of only limited self-care, confined to bed or chair more than $50 \%$ of waking hours \\
\hline 4 & Completely disabled. Cannot carry on any self-care. Totally confined to bed or chair \\
\hline 5 & Dead \\
\hline
\end{tabular}

*Reproduced courtesy of Robert Comis M.D., Group Chair, Eastern Cooperative Oncology Group; ECOGPS = Eastern Cooperative Oncology Group Performance Status 


\begin{tabular}{|c|c|}
\hline Age (yrs) & $53.1 \pm 12.4$ \\
\hline Months from echo to follow-up/death & $21.6 \pm 14.0$ \\
\hline Months from chemo to echo & $29.3 \pm 39.3$ \\
\hline Male sex & $43(36 \%)$ \\
\hline ECOGPS & $0.48 \pm 0.72$ \\
\hline Hematologic malignancy & $52(43 \%)$ \\
\hline History of anthracycline use & $69(58 \%)$ \\
\hline History of thoracic radiation & $31(26 \%)$ \\
\hline Beta blocker usage & $24(20 \%)$ \\
\hline ACE-I usage & $26(22 \%)$ \\
\hline Statin usage & $20(17 \%)$ \\
\hline Salicylate usage & $15(13 \%)$ \\
\hline Diabetes mellitus & $18(15 \%)$ \\
\hline Coronary artery disease & $4(3 \%)$ \\
\hline Hypertension & $42(35 \%)$ \\
\hline Tobacco use & $37(37 \%)$ \\
\hline Hyperlipidemia & $29(24 \%)$ \\
\hline Ejection fraction & $61.1 \pm 5.0$ \\
\hline LVEDV (mL) & $127.3 \pm 41.9$ \\
\hline IVS diastolic thickness (cm) & $1.04 \pm 0.15$ \\
\hline LVPW diastolic thickness $(\mathrm{cm})$ & $1.03 \pm 0.12$ \\
\hline Left atrial volume index $\left(\mathrm{mL} / \mathrm{m}^{2}\right)$ & $17.8 \pm 6.7$ \\
\hline Mitral E/e'** & $9.39 \pm 2.76$ \\
\hline
\end{tabular}

Values are mean \pm SD or $n(\%)$

**Mitral E/e' data interpretable in 59 survivors and 49 non-survivors

ECOGPS = Eastern Cooperative Oncology Group Performance Status; IVS = intraventricular septum;

LVPW = left ventricle posterior wall; LVEDV = left ventricle end diastolic volume 
Table 3. Breakdown of patient malignancies

\begin{tabular}{|lc|}
\hline Cancer Type & Died (\%) \\
\hline ALL & $5 / 8(63)$ \\
\hline AML & $14 / 19(74)$ \\
\hline CLL & $1 / 2(50)$ \\
\hline CML & $0 / 1(0)$ \\
\hline Hodgkin's lymphoma & $0 / 5(0)$ \\
\hline Non-Hodgkin's lymphoma & $8 / 12(67)$ \\
\hline Myelomat & $2 / 6(33)$ \\
\hline Breast Cancer & $8 / 42(19)$ \\
\hline Carcinoid Cancer & $3 / 3(100)$ \\
\hline Colorectal Cancer & $2 / 4(50)$ \\
\hline Endometrial Cancer & $0 / 1(0)$ \\
\hline Esophageal Cancer & $2 / 2(100)$ \\
\hline Head and Neck Cancers & $3 / 3(100)$ \\
\hline Lung Cancer & $5 / 3(100)$ \\
\hline Ovarian Cancer & $5 / 122(48)$ \\
\hline Pancreatic Cancer & \\
\hline Renal Cancer & \\
\hline All Cancer & \\
\hline
\end{tabular}

+Includes multiple myeloma and plasmacytoma; $\mathrm{ALL}$ = acute lymphocytic leukemia; $\mathrm{AML}$ = acute myelogenous leukemia; $\mathrm{CLL}=$ chronic lymphocytic leukemia; $\mathrm{CML}$ = chronic myelogenous leukemia 


\begin{tabular}{|c|c|c|c|c|c|c|c|c|}
\hline \multirow[b]{2}{*}{ Variables } & \multirow[b]{2}{*}{ Survived $(n=63)$} & \multirow[b]{2}{*}{ Died $(n=57)$} & \multicolumn{3}{|c|}{ Univariate } & \multicolumn{3}{|c|}{ Multivariate $\ddagger \S$} \\
\hline & & & HR & $95 \% \mathrm{Cl}$ & $p$ & HR & $95 \% \mathrm{Cl}$ & $p$ \\
\hline Age & $52.1 \pm 11.9$ & $54.3 \pm 12.9$ & 1.01 & $0.99-1.04$ & 0.255 & & & \\
\hline Time (echo to end) & $31.9 \pm 8.6$ & $10.2 \pm 8.9$ & ----- & ------------ & ---------- & & & \\
\hline Time (chemo to echo) & $32.0 \pm 41.7$ & $26.4 \pm 36.3$ & 1.00 & $0.99-1.00$ & 0.416 & & & \\
\hline Male sex & $14(22)$ & $29(51)$ & 2.26 & $1.34-3.81$ & 0.002 & 1.93 & $1.14-3.27$ & 0.014 \\
\hline ECOGPS & $0.2 \pm 0.5$ & $0.8 \pm 0.9$ & 2.16 & $1.63-2.87$ & $<0.001$ & 2.12 & $1.54-2.92$ & $<0.001$ \\
\hline Hematologic malignancy & $23(37)$ & $29(51)$ & 1.69 & $1.00-2.84$ & 0.051 & & & 0.693 \\
\hline Hx of anthracycline use & $36(57)$ & $33(58)$ & 1.13 & $0.67-1.13$ & 0.652 & & & \\
\hline Hx of thoracic radiation & $18(29)$ & $13(21)$ & 0.77 & $0.41-1.43$ & 0.408 & & & \\
\hline Beta blocker usage & $9(14)$ & $15(26)$ & 1.83 & $1.01-3.31$ & 0.046 & & & 0.130 \\
\hline ACE-I usage & $15(24)$ & $11(19)$ & 0.78 & $0.41-1.51$ & 0.465 & & & \\
\hline Statin usage & $9(14)$ & $11(19)$ & 1.31 & $0.68-2.54$ & 0.417 & & & \\
\hline Salicylate usage & $9(14)$ & $6(11)$ & 0.76 & $0.32-1.76$ & 0.515 & & & \\
\hline Diabetes mellitus & $9(14)$ & $9(16)$ & 1.24 & $0.61-2.53$ & 0.552 & & & \\
\hline Coronary artery disease & $1(2)$ & $3(5)$ & 2.24 & $0.69-7.20$ & 0.178 & & & \\
\hline Hypertension & $20(32)$ & $22(39)$ & 1.31 & $0.77-2.23$ & 0.323 & & & \\
\hline Tobacco use & $17(27)$ & $20(34)$ & 1.36 & $0.79-2.34$ & 0.273 & & & \\
\hline Hyperlipidemia & $12(19)$ & $17(30)$ & 1.41 & $0.80-2.48$ & 0.239 & & & \\
\hline Ejection fraction & $61.3 \pm 4.6$ & $60.8 \pm 5.5$ & 0.99 & $0.93-1.04$ & 0.602 & & & \\
\hline LVEDV & $129.7 \pm 41.0$ & $124.5 \pm 43.1$ & 1.00 & $0.99-1.00$ & 0.378 & & & \\
\hline IVS diastolic thickness & $1.04 \pm 0.15$ & $1.05 \pm 0.14$ & 2.05 & $0.37-11.48$ & 0.412 & & & \\
\hline LVPW diastolic thickness & $1.01 \pm 0.11$ & $1.04 \pm 0.14$ & 3.87 & $0.46-32.55$ & 0.213 & & & \\
\hline Left atrial volume index & $17.45 \pm 6.02$ & $18.17 \pm 7.61$ & 1.01 & $0.97-1.06$ & 0.645 & & & \\
\hline Mitral E/e $e^{\prime \#}$ & $9.34 \pm 2.58$ & $9.45 \pm 2.96$ & 1.02 & $0.92-1.15$ & 0.668 & & & \\
\hline GLS & $19.12 \pm 2.37$ & $17.72 \pm 3.10$ & 0.85 & $0.77-0.94$ & 0.001 & 0.89 & $0.81-0.97$ & 0.012 \\
\hline
\end{tabular}

Values are mean \pm SD or $n(\%)$ when appropriate

łusing univariate predictors with $p<0.10$ as candidate variables

$\S$ Multivariate significance defined as $p<0.05$

\#Mitral E/e' data interpretable in 59 survivors and 49 non-survivors

$\mathrm{HR}=$ hazard ratio; $\mathrm{Cl}$ = confidence interval; ECOGPS = Eastern Cooperative Oncology Group Performance Status; ACE-I = angiotensin converting enzyme inhibitor; IVS = intraventricular septum; LVPW = left ventricle posterior wall; LVEDV = left ventricle end diastolic volume; GLS = left ventricular global longitudinal systolic strain; 